\title{
A Little-Known Brain Imaging Feature in Neuromyelitis Optica Spectrum Disorder: A Leukodystrophy-Like Pattern
}

\author{
Jun Seok Lee \\ Moon Kyung Choi \\ Jae-Joon Lee \\ Hung Youl Seok \\ Department of Neurology, \\ Dongsan Medical Center \\ Keimyung University School of Medicine, \\ Daegu, Korea
}

Dear Editor,

Neuromyelitis optica spectrum disorder (NMOSD) presents with characteristic patterns of hemispheric white-matter lesions in magnetic resonance imaging (MRI), including tumefactive lesions, long spindle-like or radial-shape lesions following white-matter tracts, and asymmetric extensive and confluent hemispheric lesions. ${ }^{1}$ However, since bilateral symmetrical extensive white-matter lesions mimicking leukodystrophy have been previously reported in only three cases, ${ }^{2-4}$ its presence is not widely recognized. Here we report on a patient with anti-aquaporin-4 (anti-AQP4)-positive NMOSD who exhibited leukodystrophylike white-matter involvement.

A 67-year-old female was admitted with a 4-day history of visual loss and ocular pain in the left eye. Nine years previously she had been admitted for right optic neuritis, which was her first clinical event. At that time her visual acuity on the right side had deteriorated to hand movements, whereas it was 20/20 on the left. Brain MRI revealed only subtle hyperintensities in the left temporal deep white matter, pons, and middle cerebellar peduncles (Fig. 1AD). The presence of NMO-IgG and anti-AQP4 antibodies was not checked at that time. Her right-side visual function remained unchanged thereafter. Following the first attack, she remained stable and free of additional attacks related to demyelinating diseases of the central nervous system until the present attack, and had not been taking any medication to prevent such attacks.

At the present admission she had visual acuity of counting fingers close to the face in the left eye, while that in the right eye was similar to the previous visual acuity. There was no limb weakness, sensory disturbance, or ataxia. Her score on the Mini Mental State Examination was 28. Brain MRI performed at this time revealed bilateral symmetrical extensive hyperintensities in the subcortical and periventricular white matter, external and internal capsules, temporal pole white matter, pons, middle cerebellar peduncles, and deep cerebellar white matter (Fig. 1E-H). She was a nonsmoker and nondrinker, had no history of migraine, and had no known diabetes or other medical conditions other than hypertension. There was no family history of inherited disease. A thorough clinical history-taking and medication review revealed no clear evidence of exposure to any substances including antineoplastic agents, immunosuppressive drugs, antimicrobial agents, abused drugs, or environmental toxins, with the exception of antihypertensive medication (telmisartan/amlodipine 40/5 mg once daily). Laboratory tests searching for inherited or immune-mediated conditions such as leukodystrophies (arylsulfatase A and very-long-chain fatty acids), mitochondrial disorders (lactate and pyruvate), and Hashimoto's encephalopathy (thyroid hormone and antithyroid antibodies) all produced unremarkable findings. A cerebrospinal fluid examination showed normal cell count and protein level. Anti-AQP4 antibody was strongly positive (intensity,

(C) This is an Open Access article distributed under the terms of the Creative Commons Attribution Non-Commercial License (https://creativecommons.org/licenses/by-nc/4.0) which permits unrestricted non-commercial use, distribution, and reproduction in any medium, provided the original work is properly cited. 

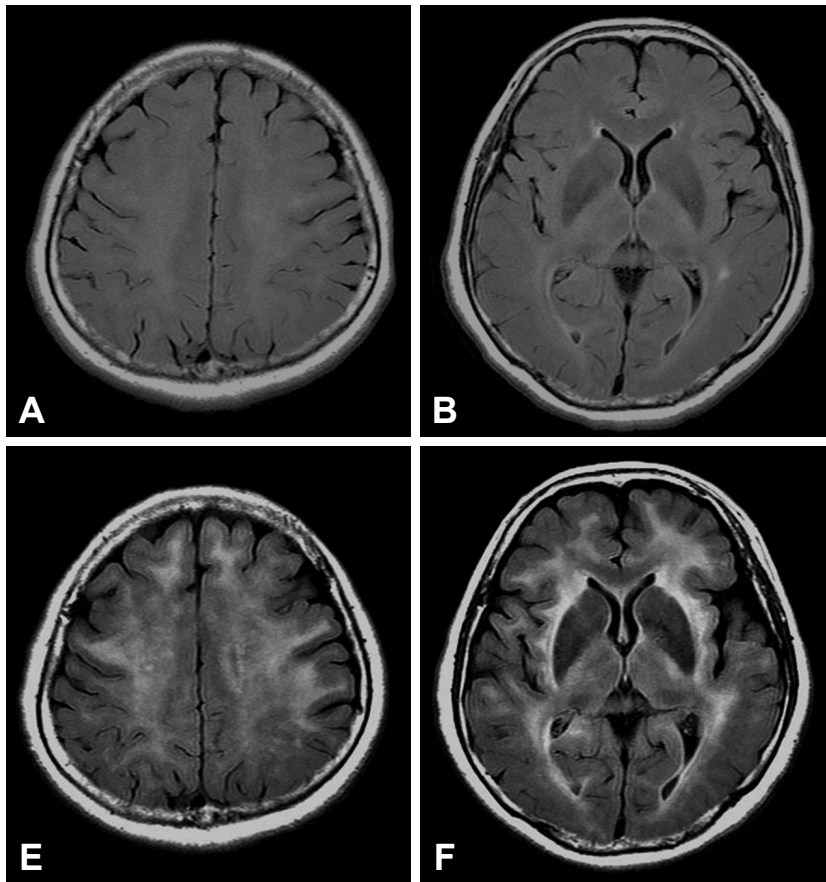
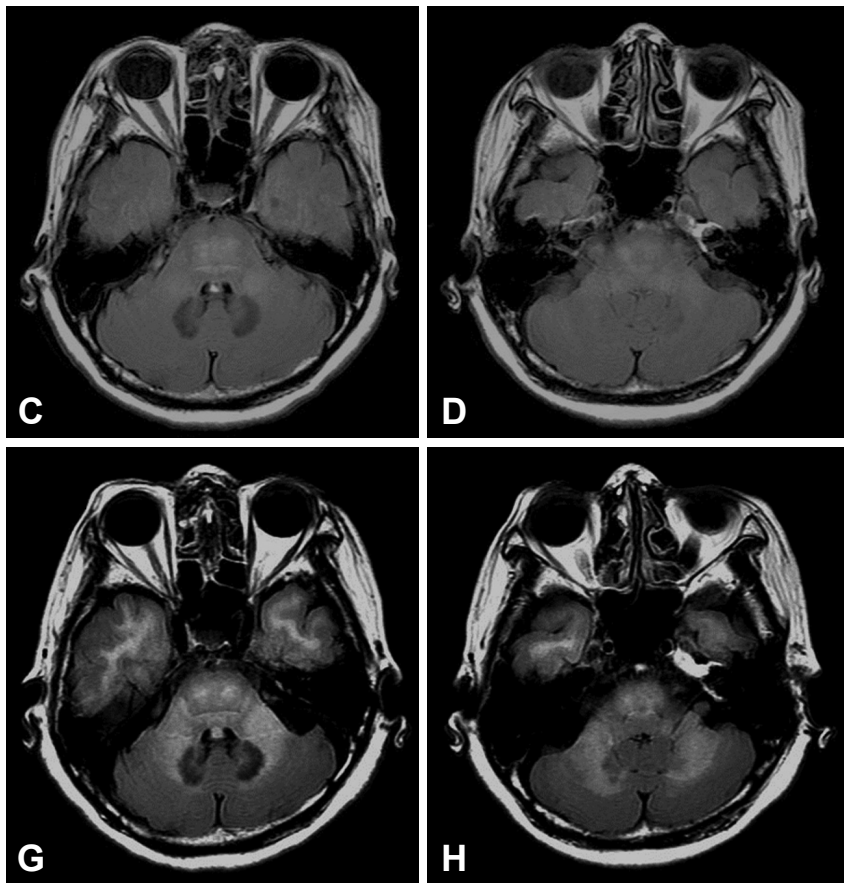

Fig. 1. MRI scans of the patient. Brain MRI performed 9 years previously (A-D) revealed only subtle hyperintensities in the left temporal deep white matter, pons, and middle cerebellar peduncle in axial T2-weighted FLAIR imaging. The present axial FLAIR images (E-H) showed bilateral symmetrical extensive hyperintensities in the subcortical and periventricular white matter, external and internal capsules, temporal pole white matter, pons, middle cerebellar peduncles, and deep cerebellar white matter. FLAIR: fluid-attenuated inversion recovery, MRI: magnetic resonance imaging.

$4+$; normal range, $1+$ to $4+$ ). She met the 2015 revised diagnostic criteria for NMOSD by having one core clinical characteristic (optic neuritis) and positivity in a test for anti-AQP4 antibody. Anti-Sjögren's syndrome-related antigen A and antiSjögren's syndrome-related antigen B antibodies were also detected. Further tests including Schirmer's test (bilaterally positive, $3 \mathrm{~mm} / 2.5 \mathrm{~mm}$ ) and salivary gland scintigraphy (decreased tracer uptake in parotid and submandibular glands) suggested the presence of Sjögren's syndrome. She was treated with intravenous methylprednisolone for 5 days at $1 \mathrm{~g}$ daily, and was then started on prednisolone to prevent further attacks. Her left visual acuity gradually improved, and there was no recurrence during her 10-month follow-up in our clinic.

The detection of anti-AQP4 antibody makes it possible to understand some MRI lesions considered typical of NMOSD. However, most of the reported MRI features of NMOSD are based on relatively short-term changes, and little is known about MRI changes in patients with long-standing NMOSD.

Our patient had anti-AQP4-positive NMOSD satisfying the 2015 revised diagnostic criteria for NMOSD. However, the less-well-known MRI changes in NMOSD resembling leukodystrophy were unexpected. In this case there was no convincing evidence for the coexistence of inherited leukodystrophies, mitochondrial disorders, and metabolic or toxic encephalopathy. On the other hand, we cannot exclude the possibility of cerebral autosomal dominant arteriopathy with subcortical infarcts and leukoencephalopathy (CADASIL) because we did not conduct the applicable genetic testing. However, the lack of a family history, the absence of clinical features suggestive of CADASIL (e.g., strokes, migraines, and dementia), and some unusual MRI findings for CADASIL (abnormalities in the deep cerebellar white matter) reduce the likelihood of CADASIL. ${ }^{5}$ Our patient had Sjögren's syndrome, which may affect the occurrence of leukodystrophy-like whitematter lesions. Three cases of NMOSD with a leukodystrophylike MRI pattern have been reported recently. ${ }^{2-4}$ One of these cases had Sjögren's syndrome, like our patient, ${ }^{3}$ but not the other two. ${ }^{2,4}$ In addition, although some studies have found cerebral white-matter lesions in Sjögren's syndrome, ${ }^{6,7}$ leukodystrophy-like white-matter changes have not been reported previously. Given that two of the three reported NMOSD cases were not associated with Sjögren's syndrome and leukodystrophy-like white-matter lesions in Sjögren's syndrome alone were not previously reported, the brain lesions of our patient were more likely to be related to current NMOSD than Sjögren's syndrome. However, the coexistence of Sjögren's syndrome may have enhanced the probability of leukodystrophic changes.

As indicated in Table 1, our case and the previous three NMOSD cases with a leukodystrophy-like MRI pattern were, overall, anti-AQP4-positive, long-standing NMOSD (disease duration $\geq 9$ years), and mainly presented with either optic 
Table 1. Summary of neuromyelitis optica spectrum disorder cases with leukodystrophy-like white-matter changes in brain MRI

\begin{tabular}{|c|c|c|c|c|c|c|c|c|c|}
\hline No. & $\begin{array}{c}\text { Sex/ } \\
\text { age, years }\end{array}$ & $\begin{array}{c}\text { Age at first/ } \\
\text { last attack, } \\
\text { years }\end{array}$ & $\begin{array}{c}\text { Disease } \\
\text { duration, } \\
\text { years }\end{array}$ & $\begin{array}{l}\text { Anti-AQP4 } \\
\text { antibody, } \\
\text { serum }\end{array}$ & $\begin{array}{c}\text { ON } \\
\text { attacks, } n\end{array}$ & $\begin{array}{l}\text { Myelitis } \\
\text { attacks, } n\end{array}$ & $\begin{array}{c}\text { Brain } \\
\text { attacks, } n\end{array}$ & $\begin{array}{l}\text { Initial brain MRI } \\
\text { (age at the scan } \\
\text { time, years) }\end{array}$ & $\begin{array}{c}\text { Other autoimmune } \\
\text { diseases }\end{array}$ \\
\hline $1^{+}$ & $\mathrm{F} / 67$ & $58 / 67$ & 9 & Positive & 2 & 0 & 0 & Nearly normal (58) & Sjögren's syndrome \\
\hline $2^{2}$ & $\mathrm{~F} / 49$ & $29 / 48$ & 20 & Positive & 20 & 4 & 0 & Normal (34) & None \\
\hline $3^{3}$ & $\mathrm{~F} / 54$ & $24 / N A$ & 30 & Positive & 1 & 1 & 0 & NA & Sjögren's syndrome \\
\hline $4^{4}$ & $\mathrm{~F} / 50$ & $14 / 50$ & 36 & Positive & 10 & 5 & $1^{*}$ & Normal (41) & None \\
\hline
\end{tabular}

*The patient had a brain attack with seizures, ${ }^{\text {t}}$ The present patient.

AQP4: aquaporin-4, F: female, MRI: magnetic resonance imaging, NA: not available, ON: optic neuritis.

neuritis or myelitis with no or only a few encephalopathy and cerebellar symptoms, despite the presence of extensive brain white-matter lesions. ${ }^{2-4}$ An especially notable point is that all of them initially showed normal or only subtle brain MRI changes, indicating that leukodystrophy-like white-matter lesions are a feature of long-standing disease. In addition, NMOSD cases with Sjögren's syndrome-constituting two out of the four cases including the current case-showed a low relapse rate compared to those without Sjögren's syndrome.

The present case has indicated that a patient with antiAQP4-positive long-standing NMOSD can exhibit MRI features similar to those of leukodystrophy. Therefore, this littleknown leukodystrophy-like MRI pattern should be considered as one brain imaging feature of long-standing NMOSD.

\section{Conflicts of Interest}

The authors have no potential conflicts of interest to disclose.

\section{Acknowledgements}

This work was supported by a National Research Foundation of Korea (NRF) grant funded by the Korea government (Ministry of Science and ICT) (No. NRF-2017R1C1B5077020).

\section{REFERENCES}

1. Kim HJ, Paul F, Lana-Peixoto MA, Tenembaum S, Asgari N, Palace J, et al. MRI characteristics of neuromyelitis optica spectrum disorder: an international update. Neurology 2015;84:1165-1173.

2. Ayrignac X, Dalière CC, Nerrant E, Vincent T, De Seze J, Labauge P. Extensive cerebral white matter involvement in a patient with NMO spectrum disorder. Mult Scler 2014;20:1401-1403.

3. Tajima Y, Yaguchi H, Mito Y. Unique central nervous system involvement and leukoencephalopathy-like magnetic resonance imaging findings in a patient with neuromyelitis optica spectrum disorder with Sjögren's syndrome. Clin Exp Neuroimmunol 2017;8:255-257.

4. Ciron J, Colin O, Rosier MP, Lapeyrie S, Biotti D, Brassat D, et al. Neuromyelitis optica spectrum disorder mimicking extensive leukodystrophy. Mult Scler 2018;24:1256-1258.

5. Stojanov D, Vojinovic S, Aracki-Trenkic A, Tasic A, Benedeto-Stojanov D, Ljubisavljevic $\mathrm{S}$, et al. Imaging characteristics of cerebral autosomal dominant arteriopathy with subcortical infarcts and leucoencephalopathy (CADASIL). Bosn J Basic Med Sci 2015;15:1-8.

6. Akasbi M, Berenguer J, Saiz A, Brito-Zerón P, Pérez-De-Lis M, Bové A, et al. White matter abnormalities in primary Sjögren syndrome. QJM 2012;105:433-443.

7. Tzarouchi LC, Zikou AK, Tsifetaki N, Astrakas LG, Konitsiotis S, Voulgari $\mathrm{P}$, et al. White matter water diffusion changes in primary Sjögren syndrome. AJNR Am J Neuroradiol 2014;35:680-685. 